ARTICLE

https://doi.org/10.1038/s41467-019-08991-8

\title{
High-resolution structure determination of sub-100 kDa complexes using conventional cryo-EM
}

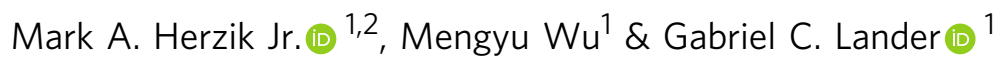

Determining high-resolution structures of biological macromolecules amassing less than 100 kilodaltons $(\mathrm{kDa})$ has been a longstanding goal of the cryo-electron microscopy (cryo-EM) community. While the Volta phase plate has enabled visualization of specimens in this size range, this instrumentation is not yet fully automated and can present technical challenges. Here, we show that conventional defocus-based cryo-EM methodologies can be used to determine high-resolution structures of specimens amassing less than $100 \mathrm{kDa}$ using a transmission electron microscope operating at $200 \mathrm{keV}$ coupled with a direct electron detector. Our $\sim 2.7 \AA$ structure of alcohol dehydrogenase $(82 \mathrm{kDa})$ proves that bound ligands can be resolved with high fidelity to enable investigation of drug-target interactions. Our $\sim 2.8 \AA$ and $~ 3.2 \AA$ structures of methemoglobin demonstrate that distinct conformational states can be identified within a dataset for proteins as small as $64 \mathrm{kDa}$. Furthermore, we provide the sub-nanometer cryo-EM structure of a sub-50 kDa protein.

\footnotetext{
${ }^{1}$ Department of Integrative Structural and Computational Biology, The Scripps Research Institute, La Jolla, CA 92037, USA. ${ }^{2}$ Present address: Department of Chemistry and Biochemistry, University of California, San Diego, La Jolla, CA 92093, USA. These authors contributed equally: Mark A. Herzik, Jr. and Mengyu Wu. Correspondence and requests for materials should be addressed to G.C.L. (email: glander@scripps.edu)
} 
$\mathrm{n}$ recent years, technical advances in cryo-electron microscopy (cryo-EM) single-particle analysis (SPA) have propelled the technique towards the forefront of structural biology, enabling the direct visualization of biological macromolecules in nearnative states at increasingly higher resolutions ${ }^{1}$. Notably, cryoEM enables three-dimensional (3D) structure determination of biological specimens in a vitrified state without the requirement of crystallization ${ }^{2}$, which has not only greatly increased the throughput of high-resolution structure determination, but has also allowed for the 3D visualization of macromolecular complexes previously deemed intractable for structural studies due to size, conformational heterogeneity, and/or compositional variability $^{3-5}$. Indeed, determining $\sim 3 \AA$ resolution reconstructions of stable, conformationally and/or compositionally homogeneous specimens by SPA has become almost routine, with an increasing number of structures at $\sim 2 \AA$ resolution or better now being reported $^{6-8}$. This resolution regime has also expanded the potential of cryo-EM SPA for structure-based drug design, particularly for targets that are less amenable to other structure determination techniques due to limited sample quantity or recalcitrance to crystallization.

Despite these advances, the radiation sensitivity of iceembedded proteins and the low signal-to-noise ratio (SNR) of cryo-EM images nonetheless impede routine structure determination for all samples, and specimen size remains a considerable limiting factor in cryo-EM SPA. Indeed, although SPA reconstructions of molecules as small as 38 kilodaltons $(\mathrm{kDa})$ have been theorized to be achievable ${ }^{9}$, this feat has yet to be realized. To date, only three macromolecular complexes smaller than $100 \mathrm{kDa}$ have been resolved to high resolution (i.e., better than $4 \AA$ ) using cryo-EM SPA: the $\sim 3.8 \AA$ resolution reconstruction of $93 \mathrm{kDa}$ isocitrate dehydrogenase ${ }^{10}$, the $\sim 3.2 \AA$ resolution reconstruction of $64 \mathrm{kDa}$ methemoglobin $(\mathrm{mHb})^{5}$, and the $\sim 3.2 \AA$ resolution reconstruction of $52 \mathrm{kDa}$ streptavidin ${ }^{11}$. Due to the limited success in imaging smaller macromolecules by cryo-EM, the technique has primarily been used to visualize large complexes, with $\sim 99 \%$ of all cryo-EM SPA reconstructions resolved to better than $5 \AA$ Å resolution comprising macromolecules amassing $>200 \mathrm{kDa}$.

We previously demonstrated that a transmission electron microscope (TEM) operating at $200 \mathrm{keV}$ equipped with a $\mathrm{K} 2$ Summit direct electron detector (DED) could be used to resolve a $\sim 150 \mathrm{kDa}$ protein complex to $\sim 2.6 \AA$ using conventional defocusbased SPA methods ${ }^{12}$. Here, we expand upon our previous results and show that biological specimens amassing $<100 \mathrm{kDa}$ can be resolved to better than $3 \AA$ resolution using similar imaging approaches. The resulting reconstructions possess well-resolved density for bound cofactors, metal ligands, as well as ordered water molecules. We further demonstrate that conformational heterogeneity in specimens of this size range can be discerned. Finally, we provide the sub-nanometer single-particle cryo-EM structure of a sub-50 kDa macromolecular complex - the $43 \mathrm{kDa}$ catalytic domain of protein kinase A.

\section{Results}

Conventional defocus-based single-particle cryo-EM. Our prior success in resolving the structure of a sub-200 $\mathrm{kDa}$ complex to better than $3 \AA$ resolution demonstrated that conventional defocus-based methodologies provided sufficient SNR to confidently assign $3 \mathrm{D}$ orientations to biological specimens that were previously thought to be too small to image ${ }^{12}$. This prompted us to investigate whether high-resolution reconstructions of macromolecules $<100 \mathrm{kDa}$ in size could be achieved with conventional imaging approaches, provided sufficiently thin ice and high particle density could be attained to maximize the accuracy of contrast transfer function estimation. All specimens in this study were imaged using a base model (i.e., excluding imaging accessories such as a phase plate or energy filter) Talos Arctica TEM equipped with a K2 Summit DED operating in counting mode using the Leginon ${ }^{13}$ automated data collection software. TEM column alignments were performed as described previously ${ }^{12,14}$ with the following modification to maximize parallel illumination: after minimizing the spread of gold powder diffraction rings, the camera length was increased to $5.7 \mathrm{~m}$ and both the size of the caustic spot and the diffraction astigmatism were iteratively minimized in order to optimize parallel illumination of the sample (see Methods).

$2.7 \AA$ resolution structure of $82 \mathrm{kDa}$ alcohol dehydrogenase. We first decided to target the $82 \mathrm{kDa}$ homodimeric enzyme alcohol dehydrogenase $(\mathrm{ADH})^{15}$, as the structure of this complex had been previously determined to near-atomic resolution (e.g., better than $1.3 \AA$ resolution) by X-ray diffraction ${ }^{16}$, providing an atomic model for validation of our results. Notably, as $\mathrm{ADH}$ is a nicotinamide adenine dinucleotide (NADH)-binding protein, part of our motivation for selecting this enzyme for structure determination was to test our ability to resolve the bound $\mathrm{NADH}$ cofactor, as this would serve as important proof-of-principle for the utility of SPA for structure-based drug design of similarly sized samples.

We further purified commercially sourced horse liver $\mathrm{ADH}$ (Sigma Aldrich) to homogeneity and prepared grids of the frozenhydrated specimen for cryo-EM data collection (see Methods) (Supplementary Fig. 1). Despite the small size of $\mathrm{ADH}$, multiple views of the homodimer could be clearly distinguished in the aligned images even when using a nominal defocus range of $-0.5 \mu \mathrm{m}$ to $-1.6 \mu \mathrm{m}$, contrary to previous estimations that significant underfocus would be required to image small $(<200$ $\mathrm{kDa}$ ) macromolecules using conventional EM ${ }^{17}$ (Supplementary Fig. 2). However, the large disparity in orthogonal dimensions of ADH $(\sim 100 \AA$ versus $\sim 40 \AA$, see Fig. 1$)$ posed a challenge during two-dimensional (2D) classification. To overcome this, two successive rounds of RELION ${ }^{18,19}$ reference-free $2 \mathrm{D}$ classification were performed, the first using a $110 \AA$ soft circular mask to identify particles comprising the longer side/tilted views, followed by a second round of $2 \mathrm{D}$ classification of the remaining data using a $60 \AA$ soft mask to identify particles comprising the end-on views (Fig. 1a, Supplementary Fig. 2e). These combined particles were subsequently subjected to several rounds of 3D autorefinement and classification with C2 symmetry enforced to yield a final $\sim 2.7 \AA$ reconstruction, as determined by gold-standard Fourier shell correlation (FSC) at $0.143^{20-22}$ (see Methods) (Fig. 1, Supplementary Table 1, and Supplementary Fig. 2). These same particles refined to $\sim 3.1 \AA$ resolution without imposing symmetry (Supplementary Fig. 2).

Inspection of the map revealed side-chain density for most of the ADH polypeptide with pronounced backbone features for the entire macromolecule. Indeed, local resolution estimates ${ }^{23}$ indicated that the majority of the map was resolved to better than $\sim 3.3 \AA$ resolution, with the core of $\mathrm{ADH}$ resolved to $\sim 2.6 \AA$ resolution (Fig. 1b). Moreover, there is unambiguous density for the bound NADH cofactor, a catalytic zinc ion within the active site of each protomer, as well as a structural zinc ion coordinated by four cysteine residues (Fig. 1). Importantly, the positions of these ligands are corroborated by the X-ray crystal structure of horse ADH (PDB ID: 2JHF [https://doi.org/10.2210/pdb2JHF/ $\mathrm{pdb}])$. This reconstruction clearly establishes that $<100 \mathrm{kDa}$ complexes can be resolved to better than $3 \AA$ resolution using conventional approaches using a $200 \mathrm{keV}$ TEM without the need for a phase plate or energy filter. Furthermore, our ability to confidently identify bound ligands and cofactors within our $\mathrm{ADH}$ 
a
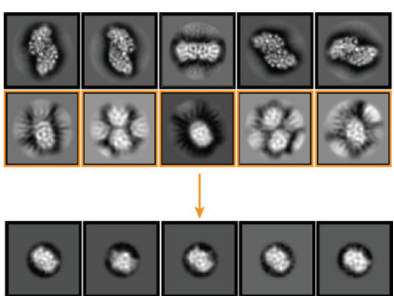

b

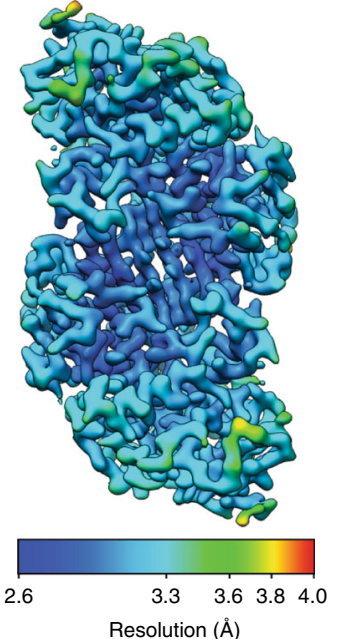

Resolution $(\AA)$ d

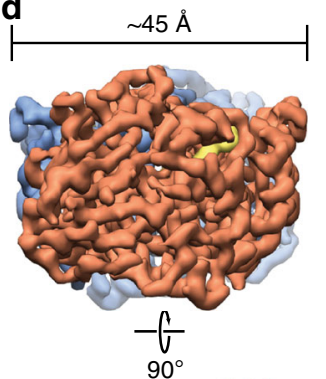

$90^{\circ}$
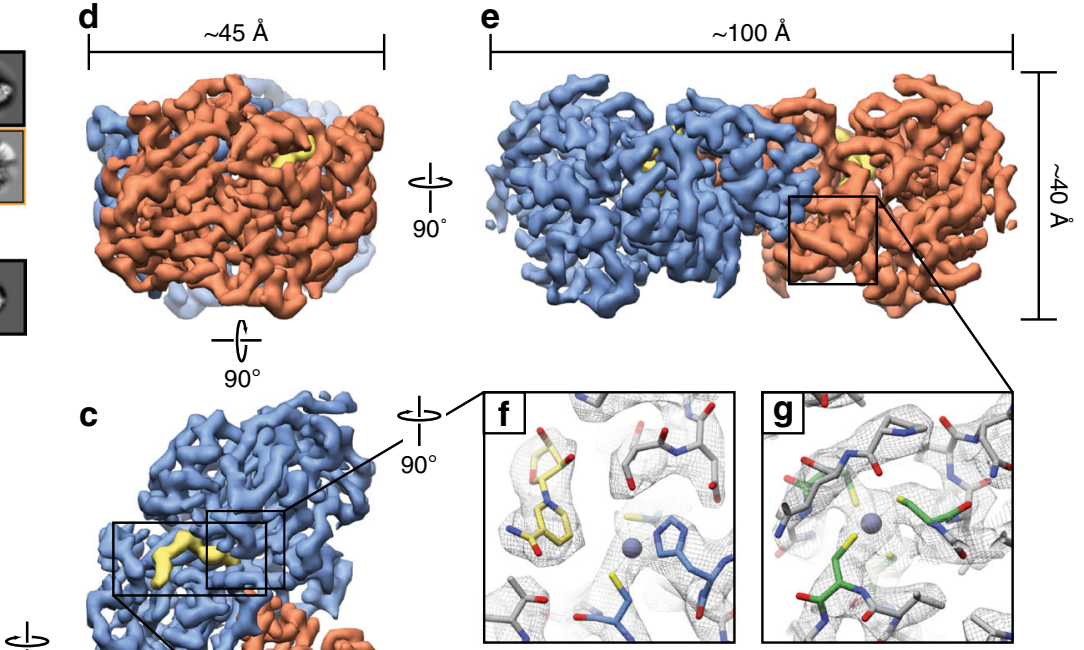

$180^{\circ}$
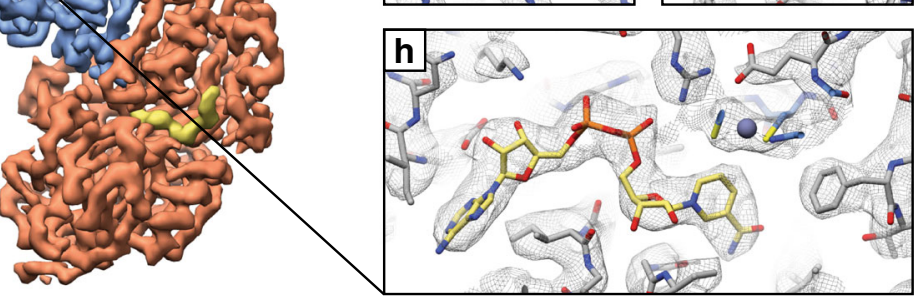

Fig. $12.7 \AA$ resolution cryo-EM reconstruction of $82 \mathrm{kDa}$ horse liver alcohol dehydrogenase. a Representative reference-free $2 \mathrm{D}$ class averages of horse liver alcohol dehydrogenase (ADH). Particles comprising the 2D classes highlighted in yellow were subsequently further classified using a smaller soft circular mask (see methods). b Final cryo-EM reconstruction colored by estimated local resolution estimated with BSOFT23. c-e Orthogonal views of the ADH EM density (colored by subunit) showing the disparity in particle dimensions. The segmented NADH EM density is shown in yellow.

f-h Zoomed-in views of the EM density (gray mesh) for the ADH active-site zinc, structural zinc site, and active site, respectively. Residues involved in coordinating the active-site zinc (blue), the structural site zinc (green) or interacting with NADH (yellow) are shown in stick representation. The zinc atoms are shown as purple spheres

EM density effectively demonstrates the utility of conventional SPA methods for structure-based drug design and other smallmolecule research involving similarly sized specimens.

Two states of methemoglobin at $\sim 2.8 \AA$ and $\sim 3.2 \AA$ resolution. Khoshouei et al. ${ }^{5}$ recently demonstrated that a TEM operating at $300 \mathrm{keV}$ combined with a Volta phase plate (VPP) and quantum energy filter could be used to resolve human hemoglobin $(\mathrm{Hb})$, a $\sim 64-\mathrm{kDa}$ heterotetrameric heme-containing protein, to $\sim 3.2 \AA$ resolution. It has been speculated that the increased SNR afforded by the VPP was integral for structure determination of $\mathrm{Hb}^{5}$. However, our ability to resolve $\mathrm{ADH}$ to high resolution indicated that conventional defocus-based imaging methodologies provided sufficient SNR for structure determination, and that we had not yet reached the size limit of this approach. We therefore sought to determine the structure of $\mathrm{Hb}$ using similar strategies. Briefly, vitrified $\mathrm{Hb}$ specimens were prepared from lyophilized human $\mathrm{Hb}$ (Sigma Aldrich) (see Methods). UV-VIS absorbance measurements of the solubilized Hb sample confirmed that the bound heme was indeed in the ferric $\left(\mathrm{Fe}^{3+}\right)$ oxidation state, hereby referred to as methemoglobin (metHb).

Images of frozen-hydrated metHb were collected similarly as described for ADH (see Methods) (Supplementary Fig. 3). Notably, orthogonal views of metHb were discernible by eye (Fig. 2b), even in micrographs imaged using underfocus values as low as $\sim 700 \mathrm{~nm}$. RELION reference-free 2D classification yielded detailed class averages exhibiting secondary-structural elements and various recognizable views of tetrameric metHb (Fig. 2c).
Three parallel 3D classifications were performed to select for unique particles comprising the best-resolved classes across each classification (see Methods, Supplementary Fig. 3). An additional round of no-alignment $3 \mathrm{D}$ classification revealed two distinct conformational states of metHb: state 1 ( $\sim 2.8 \AA$ resolution) closely matches the "Near R2" state previously described by Shibayama ${ }^{24}$ et al. 2014 (Caoot-mean-square-deviation (RMSD) $0.4 \AA$, PDB ID: 4N7P [https://doi.org/10.2210/pdb4N7P/pdb]) (Fig. 3a) and state $2(\sim 3.2 \AA$ resolution) agrees well with the "Between R and R2" state (CaRMSD 0.5 A, PDB ID: 4N7N [https://doi.org/10.2210/pdb4N7N/pdb]) (Fig. 3b). Comparison of the two states following superposition of an $\alpha \beta$ dimer from each molecule revealed an $\sim 7^{\circ}$ rigid-body rotation of one $\alpha \beta$ dimer with respect to the other with the rotation axis centered about the dimer-dimer interface, similar to those movements previously described ${ }^{24}$ (Fig. 3c). These results demonstrate that distinct, biologically relevant conformational states of a sub-100 $\mathrm{kDa}$ complex can feasibly be resolved to high-resolution using single-particle cryo-EM.

Local resolution estimates indicated that most of both maps were resolved to better than $\sim 3.5 \AA$, which is consistent with our ability to resolve side-chain densities throughout the maps (Fig. 2f). Notably, the heme pockets are estimated to be the best-resolved regions in both states (Supplementary Fig. 3), and the quality of the heme densities in both $\alpha$ and $\beta$ subunits of state 1 enabled us to confidently discern the location of the vinyl groups extending from the porphyrin ring to unambiguously assign the orientation of the heme moiety in each pocket 
a

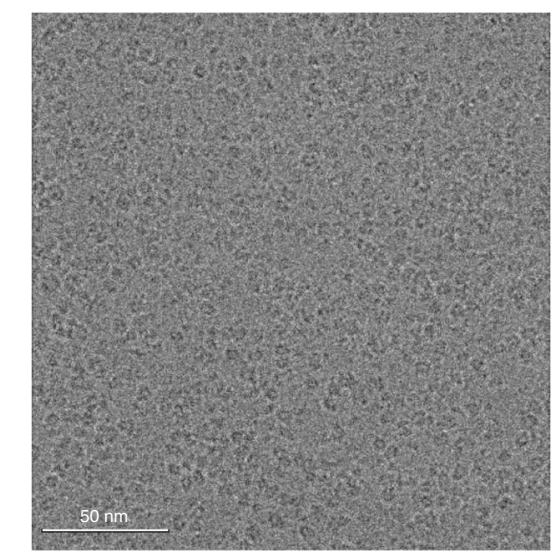

b
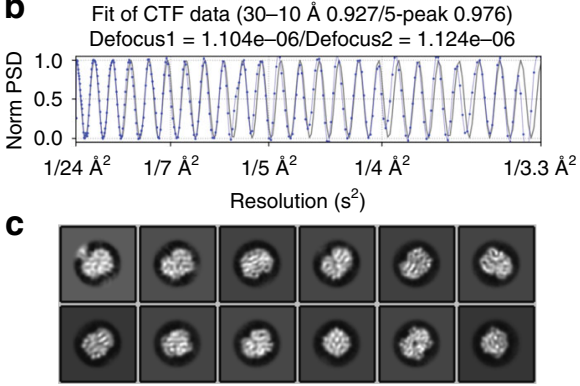

d

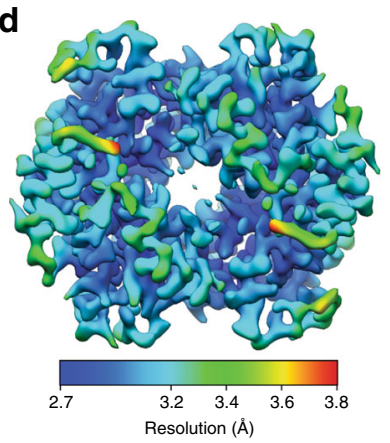

f

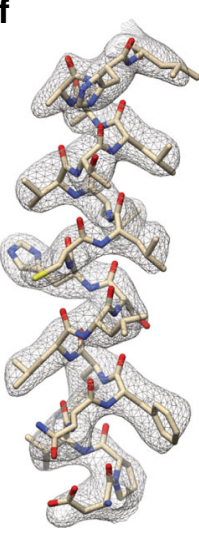

e
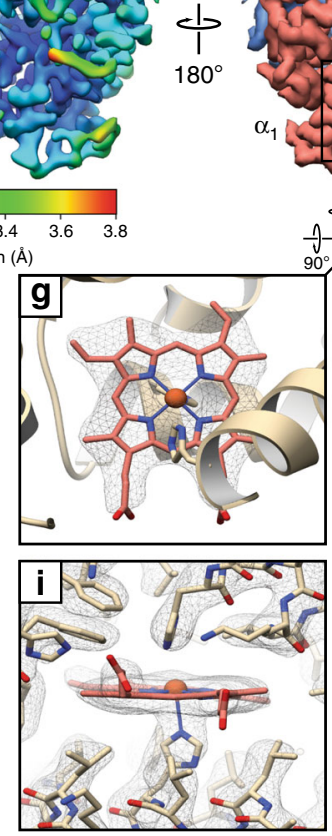
$180^{\circ}$
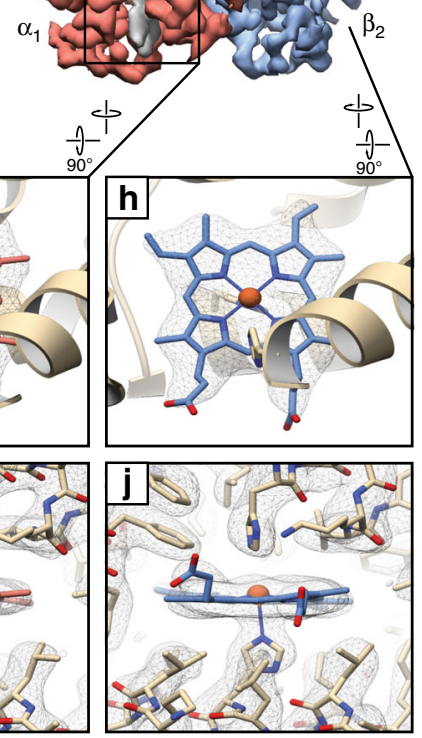

Fig. $22.8 \AA$ resolution cryo-EM reconstruction of $\sim 64 \mathrm{kDa}$ human methemoglobin. a Representative motion-corrected electron micrograph of human methemoglobin (metHb) embedded in vitreous ice recorded at $\sim 1.1 \mu \mathrm{m}$ defocus (scale bar, $50 \mathrm{~nm}$ ). b 1-dimensional plot of the contrast transfer function (CTF) Thon rings (black line) and the CTF estimated with CTFFIND4 ${ }^{33}$ (blue line). c Representative reference-free 2D class averages showing secondary structure elements. d, e Final $\sim 2.8 \AA$ resolution metHb cryo-EM density colored by local resolution (estimated using BSOFT ${ }^{23}$ ) and subunit with the segmented EM density for the heme cofactors colored gray, respectively. $\mathbf{f} E M$ density (gray mesh) zoned $2 \AA$ around an $\alpha$-helix comprising residues 94-113 from the $\alpha$ subunit. $\mathbf{g}-\mathbf{j}$ EM density in the vicinity of the heme cofactors from subunit $\alpha 1$ and $\beta 2$. Lower panels are $90^{\circ}$ rotations of upper panels. Residues are shown in stick representation (colored wheat) and the heme cofactors are colored according to the subunit coloring in e. The heme iron atoms are shown as orange spheres
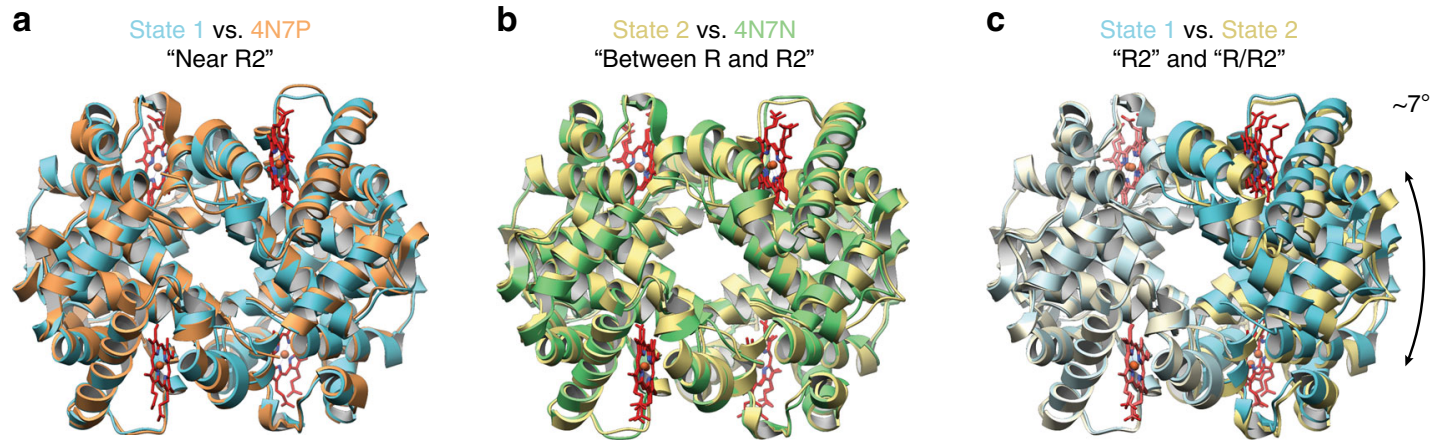

Fig. 3 Multiple conformational states of metHb determined using single-particle cryo-EM. a Cartoon representation of metHb state 1 ( $\sim 2.8 \AA$ resolution, blue) superposed with PDB ID: 4N7P [10.2210/pdb4N7P/pdb] (chains A-D, orange). b Cartoon representation of metHb state 2 ( $3.2 \AA$ resolution, yellow) superposed with PDB ID: 4N7N [10.2210/pdb4N7N/pdb] (chains E-H, green). c Superposing the $\alpha 1$ and $\beta 1$ subunits of states 1 and 2 emphasizes the difference between these two conformations as a $\sim 7^{\circ}$ rotation of the $\alpha 2$ and $\beta 2$ subunits

(Fig. 2g, h). Furthermore, the well-resolved regions of state 1 contain putative density for ordered water molecules that are conserved between those observed in a previously obtained crystal structure (PDB ID: 2DN1 [https://doi.org/10.2210/pdb2DN1/ $\mathrm{pdb}]$ ). Our ability to discern subtle conformational differences in a target as small as $64 \mathrm{kDa}$ effectively underscores the utility of this approach in examining the conformational dynamics of similarly small biological systems.
Towards a high-resolution structure of a $43 \mathrm{kDa}$ complex. Processing of the metHb dataset required two successive rounds of $2 \mathrm{D}$ classification, partially due to the presence of contaminating $\sim 32-\mathrm{kDa} \alpha \beta$ heterodimers, which surprisingly accounted for $\sim 20 \%$ of the data (Supplementary Fig. 3). Subsequent $2 \mathrm{D}$ classification of these particles yielded class averages with defined secondary-structural elements (Fig. 4). Furthermore, $2 \mathrm{D}$ projections of a simulated EM density generated using an $\alpha \beta$ 

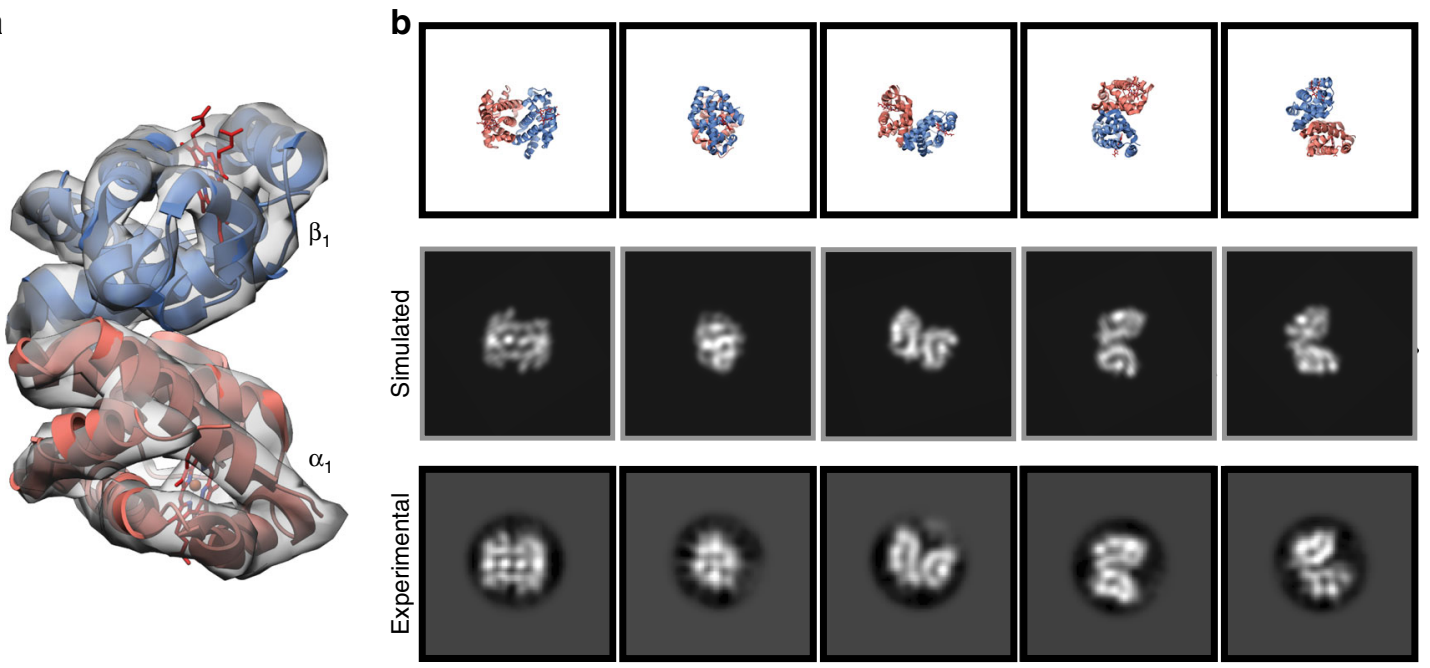

Fig. 4 2D classification yields classes of $\sim 32 \mathrm{kDa}$ hemoglobin $\alpha \beta$ heterodimer. a Simulated EM density of the hemoglobin $\alpha \beta$ heterodimer low-pass filtered to $8 \AA$ resolution shown as a gray transparent surface. The $\alpha \beta$ heterodimer atomic model is shown as ribbon cartoon and the hemes are shown as red sticks. b Views of the hemoglobin $\alpha \beta$ heterodimer atomic model (top) and 2D projections of the simulated hemoglobin $\alpha \beta$ heterodimer EM density (middle) corresponding to the class averages obtained from 2D classification (bottom) (see Fig. 2)

dimer from PDB ID: 4N7N [https://doi.org/10.2210/pdb4N7N/ pdb] exhibited striking similarity to these class averages (Fig. 4). However, generation of a $3 \mathrm{D}$ reconstruction was unsuccessful, likely due to preferred orientation and/or poor SNR of the particles in vitreous ice. Despite this, our results for the metHb tetramer indicated that we had not yet reached the size limit resolvable by our imaging methodologies, and the promising $2 \mathrm{D}$ averages of the metHb dimers prompted us to continue investigating the lower molecular weight limit of cryo-EM SPA.

We next decided to pursue the structure of the asymmetric $\sim 43 \mathrm{kDa}$ catalytic domain of protein kinase $\mathrm{A}\left(\mathrm{PKA}_{\mathrm{c}}\right)$ bound to ATP, manganese, and IP20, an inhibitory pseudo-peptide substrate that has been previously shown to stabilize $\mathrm{PKA}_{\mathrm{c}}{ }^{25}$. EM grid preparation and imaging of frozen-hydrated inhibited $\mathrm{PKA}_{\mathrm{c}}$ $\left(\mathrm{iPKA}_{\mathrm{c}}\right)$ were performed similarly as described for $\mathrm{ADH}$ and metHb with minor modifications (see Methods). Remarkably, given the small size and dimensions of $\mathrm{iPKA}_{\mathrm{c}}(65 \times 40 \times 40 \AA)$, particles were clearly discernible in the aligned micrographs even at modest underfocus values (e.g., <1000 nm) (Fig. 5).

Reference-free 2D classification of gaussian-picked particles yielded featureful $2 \mathrm{D}$ class averages representing numerous views of iPKA $\mathrm{c}_{c}$ wherein the $\mathrm{N}$ - and C-terminal lobes of the complex, as well as secondary-structural elements, could be clearly discerned. Consistent with these observations, ab initio 3D model generation using cryoSPARC ${ }^{26}$ yielded a volume resembling low-resolution iPKA $_{c}$ (Supplementary Fig. 4c). However, subsequent 3D autorefinement yielded a $>4 \AA$ resolution reconstruction of $\mathrm{iPKA}_{\mathrm{c}}$ exhibiting pronounced features consistent with preferred orientation of particles at the air-water interface (e.g., stretching along the axis orthogonal to the dominant view) and over-fitting (e.g., streaking density extending beyond the masked region), indicating that the FSC-reported resolution was substantially inflated. Examination of the Euler distribution plot confirmed that regions of Euler space were unaccounted for (Supplementary Fig. 4c). In an effort to obtain these missing views we imaged the specimen at a tilt angle of $30^{\circ}$ using methodologies previously described ${ }^{27}$ (see Methods). 2D classification of particles extracted from the tilted data gave rise to averages comprising views that were not observed in the untilted data (Fig. $5 \mathrm{c}$ ). However, the overall visual quality of the class averages from the tilted data were lower than those of the untilted data, presumably due to the combination of increased beam-induced motion and decreased SNR of the particles resulting from imaging a tilted specimen ${ }^{27}$. Nonetheless, our ability to obtain featureful iPKA ${ }_{c} 2 \mathrm{D}$ class averages from tilted micrographs indicates that this imaging scheme can be applied to biological macromolecules of a wide range of sizes.

$3 \mathrm{D}$ auto-refinement of the combined particles from the tilted and untilted data yielded a $\sim 6 \AA$ resolution reconstruction of sufficient quality to discern tertiary structure elements consistent with the $\mathrm{iPKA}_{\mathrm{c}}$ kinase fold. Although this reconstruction appeared to be more isotropic in resolution than the untilted data, the secondary structure elements of the EM density are smooth and featureless. Numerous computational efforts were employed (see Methods) to improve particle alignment and obtain a high-resolution reconstruction of $\mathrm{iPKA}_{c}$, but we were unable to overcome the resolution-limiting, gross misalignment of the particles (Supplementary Fig. 5). Given these complications arising from low image SNR, our work with $\mathrm{iPKA}_{c}$ suggests that the use of a VPP and/or energy filter may potentially benefit imaging of targets of comparably small sizes (i.e., $<50 \mathrm{kDa}$ ) by boosting observable contrast while preserving high spatial frequency information. However, the untilted 2D class averages demonstrate that detailed projections of $<50 \mathrm{kDa}$ particles are resolvable by conventional cryo-EM SPA, and that algorithmic development may enable a high-resolution structure to be produced from these data.

\section{Discussion}

The VPP has previously demonstrated excellent utility in resolving the structures of some small biological specimens ${ }^{5,11}$, and consequently is now widely perceived as a necessity for resolving smaller targets. The results presented in this study define a new frontier for target sizes that can feasibly be resolved using cryoEM without the need for a phase plate. Although the use of significant underfocus (e.g., $>2000 \mathrm{~nm}$ ) has been speculated to be required for imaging macromolecules $<200 \mathrm{kDa}$ by conventional cryo-EM SPA, it is clear from this work that even molecules as small as $\sim 43 \mathrm{kDa}$ can be resolved with modest underfocus (e.g., $<1000 \mathrm{~nm}$ ) provided the vitreous ice encompassing the molecule of interest is sufficiently thin (see Supplementary Note 1, Supplementary Fig. 6). Moreover, the results of our work with $\mathrm{ADH}$ and $\mathrm{mHb}$ demonstrate that small-molecule ligands and discrete 


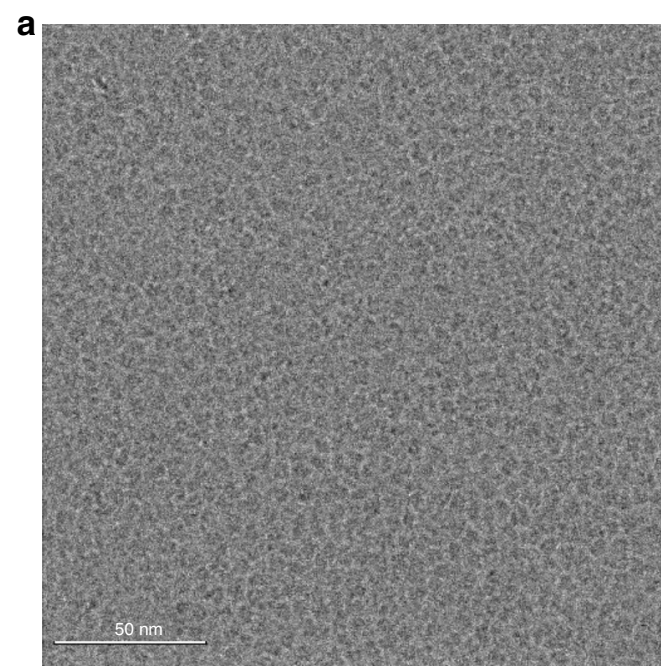

b
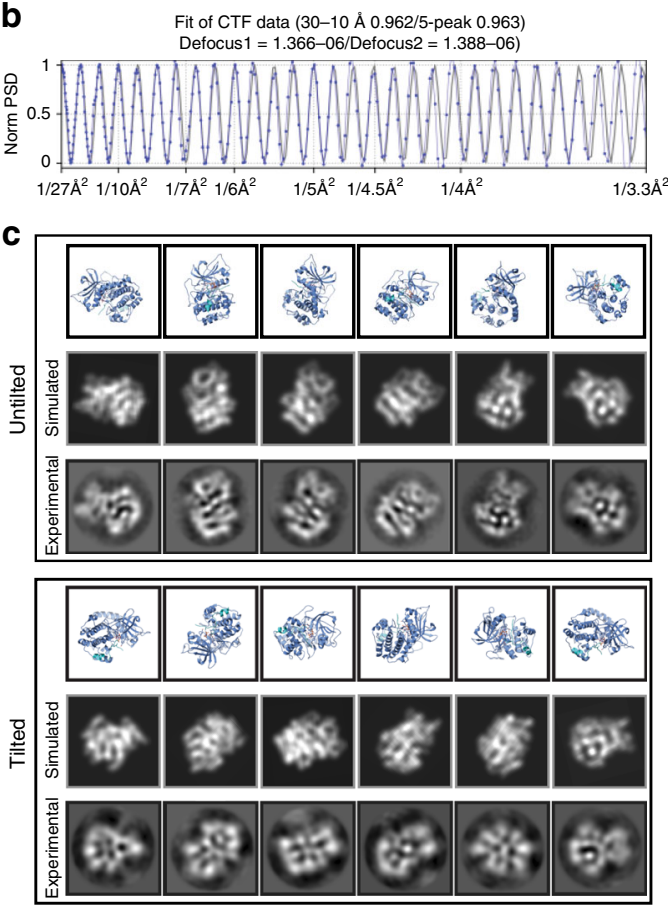

d

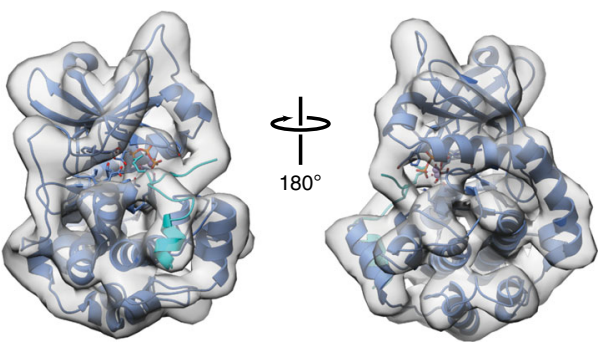

conformational states within a single sample, respectively, can be resolved using conventional SPA even for smaller complexes. Taken together, these findings broaden the potential of cryo-EM as a powerful tool for a variety of structure-based studies, particularly in drug discovery.

Though we could obtain a $3 \mathrm{D}$ reconstruction of $\mathrm{iPKA}_{\mathrm{c}}$ that allowed us to discern tertiary structural features, we were ultimately unable to achieve resolutions comparable to those of our $\mathrm{ADH}$ and $\mathrm{mHb}$ reconstructions. This was likely due to the lack of robust or accurate angular assignments of $\mathrm{iPKA}_{\mathrm{c}}$ particle images
Fig. 5 Towards a high-resolution cryo-EM reconstruction of the $\sim 43 \mathrm{kDa}$ isolable kinase domain of protein kinase A. a Representative motioncorrected electron micrograph of the catalytic subunit of protein kinase $A$

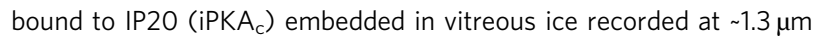
defocus (scale bar, $50 \mathrm{~nm}$ ). b 1-dimensional plot of the contrast transfer function (CTF) Thon rings (black line) and the CTF estimated with CTFFIND4 33 (blue line). c Views of the PPKA $_{c}$ atomic model (top, PDB ID: 1ATP [10.2210/pdb1ATP/pdb], blue cartoon) and 2D projections of the simulated $\mathrm{iPKA}_{c}$ EM density (middle) corresponding to the class averages obtained from 2D classification (bottom). d Final $\sim 6 \AA$ iPKA $A_{c}$ EM density shown as a transparent gray surface with the fitted atomic model (PDB ID: 1ATP [10.2210/pdb1ATP/pdb]) shown as a blue cartoon. ATP is shown as sticks and IP2O is colored light blue

arising from the inherently limited SNR of the molecule in vitreous ice that was further confounded by molecule asymmetry, manifesting as gross misalignment within the $3 \mathrm{D}$ reconstruction. It is likely that $<100 \mathrm{kDa}$ membrane proteins solubilized in detergents or nanodiscs will similarly suffer from limited SNRinduced misalignment due to the presence of the disordered molecules that surround the transmembrane domains. Future developments in detector technology and/or image-processing algorithms will be integral for high-resolution structure determination of these small protein complexes that approach the theoretical size limit of the technology. These improvements will also be of great relevance to structural studies of small membrane-embedded proteins, the SNRs for which are detrimentally impacted by the presence of disordered detergent micelles or nanodiscs. Our work further propels an important trajectory for cryo-EM SPA and indicates that high-resolution structure determination of complexes approaching, or even exceeding, the theoretical size limit of cryo-EM SPA will likely be realized in the near future.

\section{Methods}

Sample handling and protein purification. Lyophilized horse liver alcohol dehydrogenase (Sigma Aldrich) (ADH) was solubilized in $20 \mathrm{mM}$ HEPES (pH 7.5), $10 \mathrm{mM} \mathrm{NaCl}, 1 \mathrm{mM}$ TCEP (Buffer A), and dialyzed overnight against the same buffer at $4{ }^{\circ} \mathrm{C}$. Dialyzed protein was then diluted two-fold with Buffer A lacking $\mathrm{NaCl}$ and immediately loaded onto a HiTrap SP HP (GE Life Sciences) that had been equilibrated in Buffer A. ADH was eluted using a gradient from 0 to $100 \%$ Buffer B (20 mM HEPES (pH 7.5), $500 \mathrm{mM} \mathrm{NaCl,} 1 \mathrm{mM}$ TCEP) over $15 \mathrm{~mL}$ while collecting $500 \mu \mathrm{L}$ fractions. Fractions containing $\mathrm{ADH}$ were pooled and dialyzed overnight at $4{ }^{\circ} \mathrm{C}$ against $20 \mathrm{mM}$ Tris (pH 8.5), $100 \mathrm{mM} \mathrm{NaCl}, 1 \mathrm{mM}$ TCEP, and $0.5 \mathrm{mM}$ NADH (Buffer C). ADH was then concentrated to $\sim 300 \mu \mathrm{L}$ using a 30,000 MWCO spin concentrator and subjected to size-exclusion chromatography using a Superdex 10/300 GL (GE Life Sciences) that had been equilibrated in Buffer C. Pure $\mathrm{ADH}$ was pooled and concentrated to $\sim 2.5 \mathrm{mg} \mathrm{mL}^{-1}$ and used immediately for cryo-EM grid preparation.

Lyophilized human hemoglobin (Sigma Aldrich) (Hb) was solubilized in PBS ( $\mathrm{pH} 7.5$ ) to a final concentration of $\sim 12 \mathrm{mg} \mathrm{mL}^{-1}$ and used without further modifications. A UV-VIS absorbance spectrum of the solubilized protein indicated a Soret maximum at $406 \mathrm{~nm}$, consistent with previous measurements of methemoglobin (metHb), but with two small peaks in the $\alpha / \beta$ region at $576 \mathrm{~nm}$ and $541 \mathrm{~nm}$, respectively, potentially indicating a small amount of deoxyhemoglobin in the sample.

The catalytic subunit of protein kinase A (PKA) bound to ATP, manganese, and inhibitor peptide $20^{28}$ was donated by P. Aoto of the Taylor lab (UCSD) and used without modification.

Cryo-EM grid preparation. A volume of $3 \mu \mathrm{L}$ of purified $\mathrm{ADH}\left(\sim 2.5 \mathrm{mg} \mathrm{mL}^{-1}\right)$, metHb $\left(\sim 12 \mathrm{mg} \mathrm{mL}^{-1}\right)$, or inhibited PKA $\left(\sim 5 \mathrm{mg} \mathrm{mL}^{-1}\right)$ were dispensed on UltrAuFoil R1.2/1.3 300-mesh grids (Electron Microscopy Services) that had been freshly plasma cleaned using a Solarus plasma cleaner (Gatan, Inc.) with a $75 \%$ argon/25\% oxygen atmosphere at 15 Watts for $6 \mathrm{~s}$. Grids were blotted manually ${ }^{29}$ using a custom-built manual plunger in a cold room ( $\geq 95 \%$ relative humidity, $\left.4^{\circ} \mathrm{C}\right)^{12}$. Samples were blotted for $4-5 \mathrm{~s}$ with Whatman No. 1 filter paper immediately before plunge freezing in liquid ethane cooled by liquid nitrogen. We aimed to achieve a particle concentration that maximized the number of particles contained within the holes without resulting in overlapping particles and/or 
aggregation in order to maintain a consistent ice thickness across the center of holes and provide sufficient signal for accurate CTF estimation, as observed previously ${ }^{12}$.

Cryo-EM data acquisition and image processing and refinement. Microscope alignments were performed on a cross-grating calibration grid using methodologies previously described ${ }^{12,14}$ with minor modifications. Briefly, after obtaining parallel illumination in diffraction mode at a diffraction length of D $850 \mathrm{~mm}$, the length was increased to D $5.7 \mathrm{~m}$ and the intensity was adjusted to minimize the size of the caustic spot followed by minimization of the astigmatism of the diffraction lens. This process was iterated until no further improvements could be discerned visually. The resulting beam-intensity value was saved in Leginon for the exposure preset $\left(\times 73,000,0.556 \AA\right.$ pixel $\left.^{-1}\right)$ and remained unchanged throughout data collection. The objective aperture was then centered, objective lens astigmatism was minimized, and coma-free alignment was performed using Leginon ${ }^{13}$ as described previously ${ }^{12,30}$. Daily adjustments were made, if necessary, during data collection to maintain lens stigmation and to ensure the beam was centered properly. The hardware darks of the K2 Summit direct electron detector (Gatan) (DED) were updated approximately every $8-12 \mathrm{~h}$.

All cryo-EM data were collected on a Thermo Fisher Scientific Talos Arctica transmission electron microscope (TEM) operating at $200 \mathrm{keV}$. All cryo-EM data were acquired using the Leginon ${ }^{13}$ automated data collection program and all image pre-processing (e.g., frame alignment, CTF estimation, and initial particle picking) were performed in real-time using the Appion ${ }^{31}$ image-processing pipeline. Movies were collected using a K2 Summit DED operating in counting mode $\left(0.556 \AA\right.$ pixel $\left.^{-1}\right)$ at a nominal magnification of $\times 73,000$ using a defocus range of $-0.5 \mu \mathrm{m}$ to $-1.6 \mu \mathrm{m}$. Movies were collected over an $11 \mathrm{~s}$ exposure with an exposure rate of $\sim 1.95 \mathrm{e}^{-}$pixel $^{-1} \mathrm{~s}^{-1}$, resulting in a total exposure of $\sim 69 \mathrm{e}^{-} \AA^{-2}$ $\left(1.57 \mathrm{e}^{-} \AA^{-2}\right.$ frame $\left.^{-1}\right)$. Motion correction and dose-weighting were performed using the MotionCor2 frame alignment program ${ }^{32}$ as part of the Appion preprocessing workflow. Frame alignment was performed on $5 \times 5$ tiled frames with a $\mathrm{B}$-factor of $100\left(\mathrm{ADH}\right.$ and metHb) or $250\left(\mathrm{iPKA}_{\mathrm{c}}\right)$ applied. A running average of three frames was also used for $\mathrm{PRKA}_{\mathrm{c}}$ for frame alignment.

Unweighted summed images were used for CTF determination using CTFFIND4 $4^{33}$ and gCTF $^{34}$. Specifically, CTFFIND4 within Appion was used for real-time CTF determination ( 512 box size, $40 \AA$ minimum resolution, $3 \AA$ max resolution, 0.10 amplitude contrast), wherein aligned micrographs with a CTF estimate confidence of fit below $90 \%$ were eliminated from further processing. Local CTF estimates using gCTF were then performed on the remaining micrographs using the standalone package $(\mathrm{ADH})$ or a grid-based algorithm incorporated within Appion (metHb, PKA). For the latter, dummy coordinates were placed across the micrograph in rows and columns spaced 200 pixels apart and passed to gCTF for local CTF estimation using equiphase averaging (local box size of 512 pixels, 0.10 amplitude contrast, $50 \AA$ minimum resolution, $4 \AA$ max resolution, 1024-pixel field size). CTF values for a given particle were then determined using a cubic spline interpolation of the local CTF estimates within the grid.

For $\mathrm{ADH}$, difference of Gaussian (DoG) picker $^{35}$ was used to automatically pick particles from the first 145 dose-weighted micrographs yielding a stack of 135,424 picks that were binned $4 \times 4\left(2.234 \AA\right.$ pixel $^{-1}, 64$-pixel box size $)$ and subjected to reference-free $2 \mathrm{D}$ classification using RELION 2.1 ${ }^{19}$. The best five classes that represented orthogonal views of $\mathrm{ADH}$ were then used for template-based particle picking using RELION. 1,232,543 picks were extracted from 1151 dose-weighted movies, binned $4 \times 4\left(2.234 \AA\right.$ pixel $^{-1}, 64$-pixel box size $)$ and subjected to reference-free $2 \mathrm{D}$ classification using RELION using a $110 \AA$ A soft circular mask. The best $2 \mathrm{D}$ class averages that represented side or top/bottom views of $\mathrm{ADH}$ (i.e., the longest dimensions of $\mathrm{ADH}$, see Fig. 1) were then isolated (639,430 particles). Those class averages that contained "end-on" or tilted views of ADH (e.g., the smallest dimension of $\mathrm{ADH}$, see Fig. 1) were combined and subjected to another round of reference-free $2 \mathrm{D}$ classification using a $60 \AA$ soft circular mask to focus on alignment of the smaller "end-on" views. The best $2 \mathrm{D}$ class averages were then selected (20,232 particles) and combined with the previously selected particles containing the longer side views for further processing.

A total of 659,662 particles corresponding to the best 2D class averages that displayed strong secondary-structural elements and multiple views of $\mathrm{ADH}$ were selected for homogenous ab inito model generation using cryoSPARC ${ }^{26}$ to eliminate potential model bias. The generated model exhibited C2 symmetry and was low-pass filtered to $30 \AA$ for use as an initial model for $3 \mathrm{D}$ auto-refinement in RELION. 659,662 binned $4 \times 4$ particles $\left(2.234 \AA\right.$ pixel $^{-1}, 64$-pixel box size) were $3 \mathrm{D}$ autorefined into a single class followed by subsequent re-centering and re-extraction binned $2 \times 2\left(1.117 \AA\right.$ pixel $^{-1}, 256$-pixel box size). Due to the close proximity of neighboring particles, any re-centered particle within a 30-pixel range of another was considered a duplicate and subsequently removed. These particles were then $3 \mathrm{D}$ autorefined (C2 symmetry) into a single class using a scaled version of the binned $4 \times 4$ refined map. Upon convergence, the run was continued with a soft mask (5-pixel extension, 5-pixel soft cosine edge) around the entire molecule, followed by a noalignment, 3D classification (6 classes, tau_fudge $=20$ ) using the same soft mask. Particles comprising the best-resolved class was then subjected to 3D auto-refinement (C2 symmetry) using a soft mask. A subsequent no-alignment classification (2 classes, tau_fudge $=20$ ) was performed and the class that possessed the best-resolved sidechain and backbone densities were re-centered and re-extracted unbinned $(0.556 \AA$ pixel $^{-1}, 512$-pixel box size). This final stack of 11,459 particles was 3D auto-refined using a soft mask to $\sim 2.92 \AA$ (C2 symmetry) and $\sim 3.45 \AA$ (C1 symmetry) (goldstandard FSC at 0.143 cutoff $^{21}$ ). Following per-particle defocus and beam tilt refinement using RELION 3.0, the final resolution of the reconstruction improved to $\sim 2.72 \AA$ (C2 symmetry) and $\sim 3.14 \AA$ (C1 symmetry), using phase randomization to account for the convolution effects of a solvent mask on the FSC between the two independently refined half maps ${ }^{22,36}$.

For metHb, DoG picker ${ }^{35}$ was used to automatically pick particles from the first 560 dose-weighted micrographs yielding a stack of 234,895 picks that were binned $4 \times 4\left(2.234 \AA\right.$ pixel $^{-1}, 64$-pixel box size $)$ and subjected to reference-free $2 \mathrm{D}$ classification using RELION ${ }^{19}$. The best nine classes that represented orthogonal views of $\mathrm{Hb}$ were then used for template-based particle picking using RELION. $1,615,738$ picks were extracted from 1673 dose-weighted movies, binned $4 \times 4$ (2.234 $\AA$ pixel $^{-1}, 64$-pixel box size) and subjected to reference-free 2D classification using RELION using a $80 \AA$ soft circular mask. Class averages that displayed strong secondary-structural elements of metHb (513,632 particles) were combined and subjected to another round of reference-free $2 \mathrm{D}$ classification. A total of 160,169 particles corresponding to the best $2 \mathrm{D}$ class averages that displayed multiple views of tetrameric $\mathrm{Hb}$ were selected for homogenous ab inito model generation using cryoSPARC $^{26}$ to eliminate potential model bias. The generated volume was low-pass filtered to $20 \AA$ and used as an initial model for 3D auto-refinement in RELION.

Binned $4 \times 4$ particles $\left(2.234 \AA\right.$ pixel $^{-1}, 64$-pixel box size) were $3 \mathrm{D}$ auto-refined into a single class followed by subsequent re-centering and re-extraction binned $2 \times 2\left(1.117 \AA\right.$ pixel $^{-1}, 128$-pixel box size). Any re-centered particle within a 25-pixel range of another was considered a duplicate and subsequently removed. These particles were then 3D auto-refined (C2 symmetry) into a single class using a scaled version of the binned $4 \times 4$ refined map. Upon convergence, the run was continued with a soft mask (5-pixel extension, 5-pixel soft cosine edge) followed by three parallel no-alignment 3D classifications (four classes) with varying tau_fudge values $(8,12$, or 20$)$ using the same soft mask. Particles comprising the bestresolving classes across each classification were combined (35,809 particles) and duplicates were eliminated to maximize the subset of particles selected. The particles were then subjected to no-alignment classification ( 2 classes, tau_fudge $=$ $20)$. Each class was then separately refined, re-centered, and re-extracted unbinned $\left(0.556 \AA\right.$ pixel $^{-1}, 256$-pixel box size $)$ and 3D auto-refined using a soft mask. Class 1 $(24,308$ particles) refined to a final estimated resolution of $\sim 2.80 \AA$ (C2 symmetry, $\sim 3.17 \AA$ resolution with $\mathrm{C} 1$ symmetry) and Class 2 (11,501 particles) refined to a final estimated resolution of $\sim 3.25 \AA$ resolution (C2 symmetry, $\sim 3.48 \AA$ resolution with $\mathrm{C} 1$ symmetry) according to gold-standard $\mathrm{FSC}^{21}$ using phase randomization to account for the convolution effects of a solvent mask on the FSC between the two independently refined half maps ${ }^{22,36}$. Per-particle defocus and beam tilt refinement using RELION 3.0 did not yield improvements to the reconstructions.

Local resolution estimates for all reconstructions were calculated using the bloc_res function in BSOFT ${ }^{23}$.

For PKA, DoG picker ${ }^{35}$ was used to automatically pick particles from the untilted, aligned and dose-weighted micrographs yielding a stack of 554,170 picks that were binned $4 \times 4\left(2.234 \AA\right.$ pixel $^{-1}, 64$-pixel box size $)$ and subjected to reference-free $2 \mathrm{D}$ classification using $\mathrm{RELION}^{19}$. A total of 314,001 particles corresponding to the best $2 \mathrm{D}$ class averages that displayed multiple views of iPKA were selected for homogenous ab inito model generation using cryoSPARC ${ }^{26}$ to eliminate potential model bias. The generated volume was low-pass filtered to $20 \AA$ and used as an initial model for 3D auto-refinement (C1 symmetry) in RELION. Particles were then re-centered and re-extracted, Fourier binned $2 \times 2(1.117 \AA$ / pixel, 128-pixel box) and subsequently $3 \mathrm{D}$ classified (tau_fudge $=4$, E-step limit $=$ $7 \AA$ ). Particles comprising the best-resolved class was 3D auto-refined to $\sim 4.26 \AA$ resolution, as estimated by gold-standard FSC. However, this EM density exhibited artifacts associated with preferred orientation (stretched features in directions orthogonal to the preferred view) and did not possess high-resolution features consistent with the Gold-standard FSC-estimated resolution of $\sim 4.26 \AA$ resolution. In an attempt to obtain the missing views of $\mathrm{iPKA}_{c}$ and potentially improve the resolution of our reconstruction we collected a dataset at a tilt angle of $30^{\circ}$ using methodologies previously described ${ }^{27}$. These aligned, dose-weighted micrographs were combined with the untilted data and forward projections of a $10 \AA$ low-pass filtered EM density of $\mathrm{iPKA}_{\mathrm{c}}$ were used for template-based particle picking within RELION. Together, 1,762,088 particles were extracted, Fourier binned $4 \times 4$ (2.234 ̊̊/pixel, 64-pixel box), and subjected to reference-free 2D classification (tau_fudge $=1$, E-step limit $=7 \AA$ ) using RELION. It was apparent from the resulting $2 \mathrm{D}$ class averages that additional views of $\mathrm{iPKA}_{\mathrm{c}}$ were obtained from imaging a tilted specimen (Fig. 5). Particles comprising the "best" classes were 3D classified (tau_fudge $=1$, E-step limit $=7 \AA$ ) and those classes that most resembled $\mathrm{iPKA}_{\mathrm{c}}$ were subjected to an additional round of $3 \mathrm{D}$ classification (tau_fudge $=1$, E-step limit $=7 \AA$ ). The "best" resolved classes were then $3 \mathrm{D}$ auto-refined (C1 symmetry) to yield a $\sim 4.57 \AA$ resolution reconstruction, as estimated by goldstandard FSC. Although these particles refined to a similar resolution as the untitled data alone, the resulting reconstruction was more isotropic. Particles were recentered and re-extracted, Fourier binned $2 \times 2$ (1.117 $\AA$ /pixel, 128-pixel box $)$ and duplicate particle picks were eliminated. These particles were $3 \mathrm{D}$ auto-refined to $\sim 6.24 \AA$ resolution and subjected to no-alignment 3D classification (tau_fudge $=6$ ). The "best" resolved classes were combined and 3D auto-refined to $\sim 6.17 \AA$ resolution. Refined particles with NrOfSignificantSamples values $>25$ were eliminated and the remaining particles were 3D auto-refined to $\sim 4.34 \AA$ resolution (as estimated by gold-standard FSC). 
Although the additional views from the tilted dataset aided in lessening the artifacts resulting from preferred orientation, the final EM density does not possess molecular features consistent with the reported resolution of $\sim 4.3 \AA$ but rather resembles a $\sim 6-7 \AA$ resolution EM density. Strategies to further improve the quality of the reconstruction did not yield significant results. Such attempts included: (1) high-pass filtering the extracted particles to 100 or $120 \AA$ prior to 3D refinements as has previously been implemented for high-resolution structure determination of small proteins ${ }^{11}$; (2) increasing the tau2_fudge value (e.g., 4, 6, 8, etc.) during refinement to place greater weight on the experimental data; (3) increasing the amplitude contrast of the inputted particle stack (e.g., 0.20 vs. 0.10 as used for the final structure); (4) filtering the particles iteratively by RELION metadata metrics (e.g., NrOfSignificantSamples, MaxValueProbDistribution, or Z-score) (5) using particle images with a box size just outside the bounds of the particle (e.g., 72 pixel box vs. 128 -pixel box for binned $2 \times 2$ particles); or (6) various combinations of the above mentioned.

Model building and refinement. For each of the final reconstructions, an initial model that had been stripped of all ligands, waters, and alternative conformations, had all occupancies set to zero, had a single B-factor value was set for all atoms, and had all Ramachandran, rotameric, and geometric outliers corrected, was subjected to a multi-model pipeline using methodologies similar to those previously described ${ }^{37}$. Briefly, PDB ID: 2JHF [https://doi.org/10.2210/pdb2JHF/pdb] was used as the starting model for ADH, chains A-D from PDB ID: 4N7P [https://doi. org/10.2210/pdb4N7P/pdb] was used for metHb class 1, and chains E-H from PDB ID: 4N7N [https://doi.org/10.2210/pdb4N7N/pdb] were used for metHb class 2. These initial models were then refined into the EM density using Rosetta ${ }^{38}$ while enforcing the same symmetry that was applied during reconstruction and adjusting the Rosetta weighting and scoring functions according to the FSC-estimated map resolution. Each of the 200 Rosetta-refined models were then ranked based on the number of Ramachandran outliers, geometry violations, Rosetta aggregate score, and MolProbity clashscore ${ }^{39}$. The 10 structures that scored the best across all categories were selected for further real-space refinement using the Phenix refinement package ${ }^{40}$ after incorporating cofactors (e.g., NADH for $\mathrm{ADH}$ and heme for metHb) and active-site and structural $\mathrm{Zn}$ ions (ADH). Model-model agreement statistics are based on the per-residue Ca RMSD ${ }^{37}$.

Reporting summary. Further information on experimental design is available in the Nature Research Reporting Summary linked to this article.

\section{Data availability}

Data supporting the findings of this manuscript are available from the corresponding author upon reasonable request. A reporting summary for this Article is available as a Supplementary Information file. The atomic coordinates for the ADH and metHb (class 1 and class 2) structures have been deposited in the Protein Data Bank (PDB) under accession codes $6 \mathrm{NBB}$ [https://doi.org/10.2210/pdb6NBB/pdb], 6NBC [https://doi.org/ 10.2210/pdb6NBC/pdb], and 6NBD [https://doi.org/10.2210/pdb6NBD/pdb], respectively. The corresponding EM density maps (final unsharpened and sharpened maps, half maps, and masks) have been deposited to the Electron Microscopy Data Bank under accessions EMD-0406, EMD-0407 and EMD-0408, respectively, and EMD-0409 for $\mathrm{iPKA}_{\mathrm{c}}$. Uncorrected movie frames and associated gain correction images for ADH, metHb, and $\mathrm{PPKA}_{c}$ datasets are available on the EMPIAR under accessions 10249, 10250, and 10252 , respectively.

Received: 5 December 2018 Accepted: 11 February 2019

Published online: 04 March 2019

\section{References}

1. Cheng, Y. Single-particle cryo-EM-how did it get here and where will it go. Science 361, 876-880 (2018).

2. Nogales, E. \& Scheres, S. H. W. Cryo-EM: a unique tool for the visualization of macromolecular complexity. Mol. Cell 58, 677-689 (2015).

3. Lander, G. C. et al. Complete subunit architecture of the proteasome regulatory particle. Nature 482, 186-191 (2012).

4. Yan, C., Wan, R., Bai, R., Huang, G. \& Shi, Y. Structure of a yeast activated spliceosome at $3.5 \AA$ resolution. Science 353, 904-911 (2016).

5. Khoshouei, M., Radjainia, M., Baumeister, W. \& Danev, R. Cryo-EM structure of haemoglobin at $3.2 \AA$ determined with the Volta phase plate. Nat. Commun. 8, 16099 (2017).

6. Bartesaghi, A. et al. $2.2 \AA$ resolution cryo-EM structure of $\beta$-galactosidase in complex with a cell-permeant inhibitor. Science 348, 1147-1151 (2015).

7. Bartesaghi, A. et al. Atomic resolution cryo-EM structure of $\beta$-galactosidase. Structure 26, 848-856.e3 (2018).

8. Tan, Y. Z. et al. Sub-2 ̊̊ Ewald curvature corrected structure of an AAV2 capsid variant. Nat. Commun. 9, 3628 (2018).
9. Henderson, R. The potential and limitations of neutrons, electrons and X-rays for atomic resolution microscopy of unstained biological molecules. Q. Rev. Biophys. 28, 171-193 (1995).

10. Merk, A. et al. Breaking cryo-EM resolution barriers to facilitate drug discovery. Cell 165, 1698-1707 (2016).

11. Fan, X. et al. Single particle cryo-EM reconstruction of $52 \mathrm{kDa}$ streptavidin at 3.2 Angstrom resolution. Preprint at, https://doi.org/10.1101/457861 (2018).

12. Herzik, M. A., Wu, M. \& Lander, G. C. Achieving better-than-3- $\AA$ resolution by single-particle cryo-EM at $200 \mathrm{keV}$. Nat. Methods 14, 1075-1078 (2017).

13. Suloway, C. et al. Automated molecular microscopy: the new Leginon system. J. Struct. Biol. 151, 41-60 (2005).

14. Herzik Mark, A. J., Wu, M. \& Lander, G. C. Setting up the Talos Arctica electron microscope and Gatan K2 direct detector for high-resolution cryogenic single-particle data acquisition, https://doi.org/10.1038/ protex.2017.108 (2017).

15. Eklund, H. et al. The structure of horse liver alcohol dehydrogenase. FEBS Lett. 44, 200-204 (1974).

16. Meijers, R. et al. Structural evidence for a ligand coordination switch in liver alcohol dehydrogenase. Biochemistry 46, 5446-5454 (2007).

17. Cheng, Y., Grigorieff, N., Penczek, P. A. \& Walz, T. A primer to single-particle cryo-electron microscopy. Cell 161, 438-449 (2015).

18. Scheres, S. H. W. RELION: implementation of a Bayesian approach to cryoEM structure determination. J. Struct. Biol. 180, 519-530 (2012).

19. Kimanius, D., Forsberg, B. O., Scheres, S. H. \& Lindahl, E. Accelerated cryoEM structure determination with parallelisation using GPUs in RELION-2. Elife 5 (2016).

20. Henderson, R. et al. Outcome of the first electron microscopy validation task force meeting. Structure 20, 205-2014 (2012).

21. Scheres, S. H. W. \& Chen, S. Prevention of overfitting in cryo-EM structure determination. Nat. Methods 9, 853-854 (2012).

22. Chen, S. et al. High-resolution noise substitution to measure overfitting and validate resolution in $3 \mathrm{D}$ structure determination by single particle electron cryomicroscopy. Ultramicroscopy 135, 24-35 (2013).

23. Cardone, G., Heymann, J. B. \& Steven, A., . \& Steven, C. One number does not fit all: Mapping local variations in resolution in cryo-EM reconstructions. $J$. Struct. Biol. 184, 226-236 (2013).

24. Shibayama, N., Sugiyama, K., Tame, J. R. H. \& Park, S.-Y. Capturing the hemoglobin allosteric transition in a single crystal form. J. Am. Chem. Soc. 136, 5097-5105 (2014).

25. Moore, M. J., Adams, J. A. \& Taylor, S. S. Structural basis for peptide binding in protein kinase A. Role of glutamic acid 203 and tyrosine 204 in the peptidepositioning loop. J. Biol. Chem. 278, 10613-10618 (2003).

26. Punjani, A., Rubinstein, J. L., Fleet, D. J. \& Brubaker, M. A. cryoSPARC: algorithms for rapid unsupervised cryo-EM structure determination. Nat. Methods 14, 290-296 (2017).

27. Tan, Y. Z. et al. Addressing preferred specimen orientation in single-particle cryo-EM through tilting. Nat. Methods 14, 793-796 (2017).

28. Zheng, J. et al. $2.2 \AA$ A refined crystal structure of the catalytic subunit of cAMPdependent protein kinase complexed with MnATP and a peptide inhibitor. Acta Crystallogr. D. Biol. Crystallogr. 49, 362-365 (1993).

29. Dubochet, J. et al. Cryo-electron microscopy of vitrified specimens. Q. Rev. Biophys. 21, 129-228 (1988)

30. Glaeser, R. M., Typke, D., Tiemeijer, P. C., Pulokas, J. \& Cheng, A. Precise beamtilt alignment and collimation are required to minimize the phase error associated with coma in high-resolution cryo-EM. J. Struct. Biol. 174, 1-10 (2011).

31. Lander, G. C. et al. Appion: an integrated, database-driven pipeline to facilitate EM image processing. J. Struct. Biol. 166, 95-102 (2009).

32. Zheng, S. Q. et al. MotionCor2: anisotropic correction of beam-induced motion for improved cryo-electron microscopy. Nat. Methods 14, 331-332 (2017).

33. Rohou, A. \& Grigorieff, N. CTFFIND4: fast and accurate defocus estimation from electron micrographs. J. Struct. Biol. 192, 216-221 (2015).

34. Zhang, K. Gctf: Real-time CTF determination and correction. J. Struct. Biol. 193, 1-12 (2016).

35. Voss, N. R., Yoshioka, C., Radermacher, M., Potter, C. S. \& Carragher, B. DoG Picker and TiltPicker: software tools to facilitate particle selection in single particle electron microscopy. J. Struct. Biol. 166, 205-213 (2009).

36. Rosenthal, P. B. \& Henderson, R. Optimal determination of particle orientation, absolute hand, and contrast loss in single-particle electron cryomicroscopy. J. Mol. Biol. 333, 721-745 (2003).

37. Herzik Mark, A., Fraser, J. S. \& Lander, G. C. A multi-model approach to assessing local and global cryo-EM map quality. Structure 27, 344-358 (2019).

38. Wang, R. Y.-R. et al. Automated structure refinement of macromolecular assemblies from cryo-EM maps using Rosetta. Elife 5 (2016).

39. Chen, V. B. et al. MolProbity: all-atom structure validation for macromolecular crystallography. Acta Crystallogr. D. Biol. Crystallogr. 66, 12-21 (2010).

40. Adams, P. D. et al. PHENIX: a comprehensive Python-based system for macromolecular structure solution. Acta Crystallogr. D. Biol. Crystallogr. 66, 213-221 (2010) 


\section{Acknowledgements}

We thank J.C. Ducom at The Scripps Research Institute (TSRI) High Performance Computing facility for computational support, B. Anderson at the TSRI electron microscopy facility for microscope support, and M. Vos for advice and discussion regarding TEM alignments. We thank P. Aoto of the S. Taylor laboratory (University of California, San Diego) for kindly providing PRKA $_{c}$ for this study. G.C.L. is supported as a Searle Scholar and as a Pew Scholar, by a young investigator award from Amgen, and by the US National Institutes of Health (NIH) grant DP2EB020402. Computational analyses of EM data were performed using shared instrumentation at TSRI funded by NIH S10OD021634. M.A.H. is supported by a Helen Hay Whitney Foundation postdoctoral fellowship. M.W is supported by a National Science Foundation Graduate Student Research Fellowship.

\section{Author contributions}

M.A.H. and M.W. performed all cryo-EM experiments and analyses under the supervision of G.C.L., M.A.H., M.W., and G.C.L. contributed to the experimental design and manuscript preparation.

\section{Additional information}

Supplementary Information accompanies this paper at https://doi.org/10.1038/s41467019-08991-8.

Competing interests: The authors declare no competing interests.
Reprints and permission information is available online at http://npg.nature.com/ reprintsandpermissions/

Journal peer review information Nature Communications thanks Arne Moeller, Radostin Danev and Hong-Wei Wang for their contribution to the peer review of this work. Peer reviewer reports are available.

Publisher's note: Springer Nature remains neutral with regard to jurisdictional claims in published maps and institutional affiliations.

\section{(c) (i)}

Open Access This article is licensed under a Creative Commons Attribution 4.0 International License, which permits use, sharing, adaptation, distribution and reproduction in any medium or format, as long as you give appropriate credit to the original author(s) and the source, provide a link to the Creative Commons license, and indicate if changes were made. The images or other third party material in this article are included in the article's Creative Commons license, unless indicated otherwise in a credit line to the material. If material is not included in the article's Creative Commons license and your intended use is not permitted by statutory regulation or exceeds the permitted use, you will need to obtain permission directly from the copyright holder. To view a copy of this license, visit http://creativecommons.org/licenses/by/4.0/.

(C) The Author(s) 2019 